\title{
Design of electrostatic actuators for suppressing vertical disturbances of CMOS-MEMS capacitive force sensors in bio applications
}

\author{
Reza Jalil Mozhdehi ${ }^{a}$, Ali Selk Ghafari and Amir A.A. Khayyat \\ Department of Mechatronics Engineering, Sharif University of Technology, International Campus, \\ Amir Kabir Square, Kish, Iran
}

Received 26 August 2014, Accepted 23 December 2014

\begin{abstract}
The objective of this work is to design electrostatic actuators for a CMOS-MEMS nano-newton capacitive force sensor to suppress vertical vibrations disturbances. Electrostatic actuators are selected because the movable part of this force sensor is anchored to the fixed parts. In the first step, we propose a framework for simulation of the force sensor based on finite element method. The proposed model is modified utilizing comparison between the simulation and experimental models to improve the performance of the model. Then, 14 pairs of electrostatic actuators are designed for applying the control algorithm and their pull-in voltage is calculated. In next step, Modal Analysis is applied to find dominant natural frequencies and mode shape vectors. In addition, an observer is proposed to estimate the velocity of the modal coordinate. Finally, an optimal controller is designed employing state-space approach to suppress vertical vibration due to undesired out-of-plane excitations generated by environment during manipulation. Simulation results illustrate that employing optimum LQR control approach, the maximum out-of-plane disturbance input is suppressed less than $0.4 \mathrm{~s}$ with acceptable range of voltage less than pull-in voltage.
\end{abstract}

Key words: CMOS-MEMS / electrostatic actuators / modal analysis / LQR controller / observer

\section{Introduction}

MEMS force sensing devices play an important role in sensitive applications such as living cell manipulation and minimally invasive surgery (MIS). Determining forces accurately is the key point to independently manipulate vulnerable biological cells and maintain safety during manipulation.

Various kinds of MEMS force sensors have been developed and presented in the literature. A MEMS lateral force sensor was presented for a diagnostic biomechanics platform by Susan [1]. The proposed force sensor is highly sensitive and it can be directly submerged in cell medium to operate at a low voltage. Nevertheless, lateral sensing mechanism is the most problematic disadvantage of this sensor. Impairing effects of in-plane sensing mechanism are pull-in effect and complicated electrical isolation [2]. Also, a 6-DOF force and torque sensor applied for micro-manipulation applications was proposed by Estevez et al. [3]. The sensor has the capability of detecting

\footnotetext{
a Corresponding author:

r.jalilmozhdehi@sharif.kish.ac.ir
}

forces and torques in $\mu \mathrm{N}$ range. However, in minimally invasive surgery and living cell manipulation the amplitude of the forces may be varied in nano-newton range. The piezoelectric [4], optical [5], piezoresistive [6] and capacitive force sensors are the most important types offered for this purpose. Chu et al. developed a highly sensitive capacitive force sensor in 2007 [7]. The main disadvantages of this sensor are in-plane sensing mechanism and its structural integration with a gripper. To resolve mentioned drawbacks novel COMS- MEMS technology was proposed for fabrication. Advantages of this technology are high performance, small size and cost effectiveness. Other advantages are better integration with gripper and integration of multiple sensors into one platform [2]. This method was used for fabrication of accelerometers such as the accelerometer developed by Xie and Fedder [8]. Later, a CMOS-MEMS accelerometer with tri-axis sensing electrodes arrays was proposed by Tsai et al. [9]. In addition, Xie and Fedder developed a CMOS-MEMS sensingactuating system equipped with vertical capacitive comb fingers [10]. The Nano-Newton CMOS-MEMS capacitive force sensor developed in Khir et al. [2] has utilized out-of-plane sensing mechanism with high accuracy and 


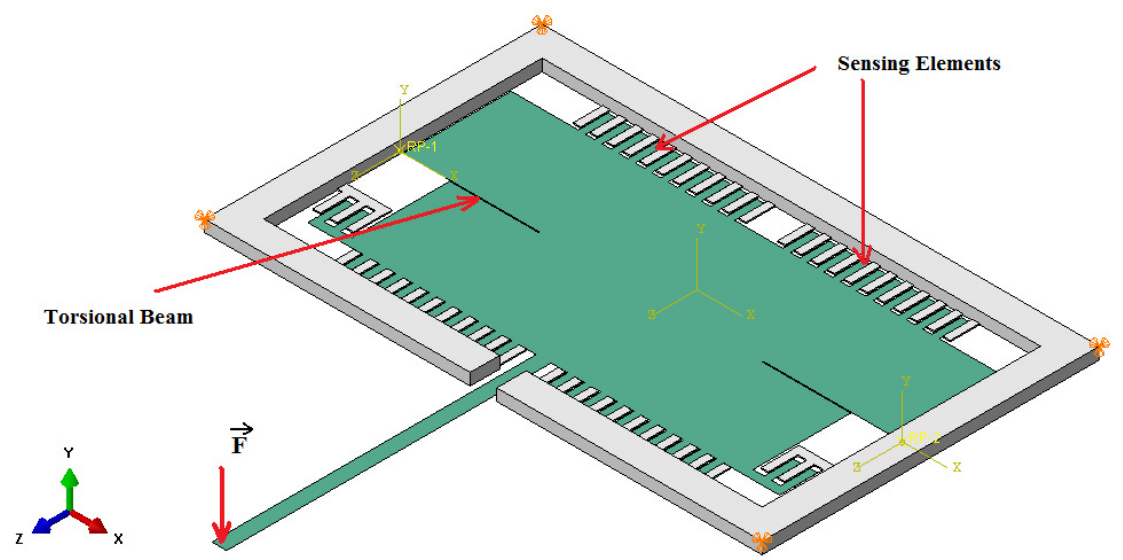

Fig. 1. A CAD model of the CMOS-MEMS nano-newton force sensor used in this research.

reliability. This force sensor can measure forces ranged in nano scale which can be useful for bio application. Furthermore, CMOS-MEMS technology is used to fabricate this force sensor that causes better integration of the sensor with manipulator. The most important characteristics of being capacitive are relatively high sensitivity and low temperature dependence [7].

One of most reasonable approach to increase the accuracy in MEMS force sensors is to eliminate disturbances and undesired forces during manipulation. Since the magnitude of sensing force during manipulation in these devices is limited to nano-newton scale, even small undesired forces can influence on the performance of the sensor severely. Most of the sensors in biomedical applications are used usually in an open loop sense in presence of different environments. In practical point of view, undesired forces applied during manipulation cannot be suppressed and may affect the accuracy and reliability of the measuring device. To suppress undesired effects of the disturbance forces using additional actuating elements in the sensor structure with a closed loop strategy seems to be an acceptable solution. There are different kinds of actuators for controlling undesired vibrations of MEMS devices such as shape memory alloy, PZT, capacitive, and etc. The position control in MEMS shape memory alloys and their nonlinear thermo mechanical behavior were investigated by Kumar et al. [11]. Gedouin et al. compared the results of a classical PID and model-free control on position control of a shape memory alloy in 2011 [12]. Marinaki et al. applied a piezoelectric sensors and actuators system for suppressing disturbances in 2011 [13]. Also, Koha investigated on single s-shaped PZT as actuating system in 2011 [14].

The main focus of this research is to propose electrostatic actuators for the structure of CMOS-MEMS NanoNewton capacitive force sensor developed in [2]. For this purpose, the model proposed for the force sensor is simulated by finite element method (FEM). Then 14 pairs of electrostatic actuators are proposed and their pull-in voltage is calculated. Electrostatic actuators are selected because the movable part of this force sensor is anchored

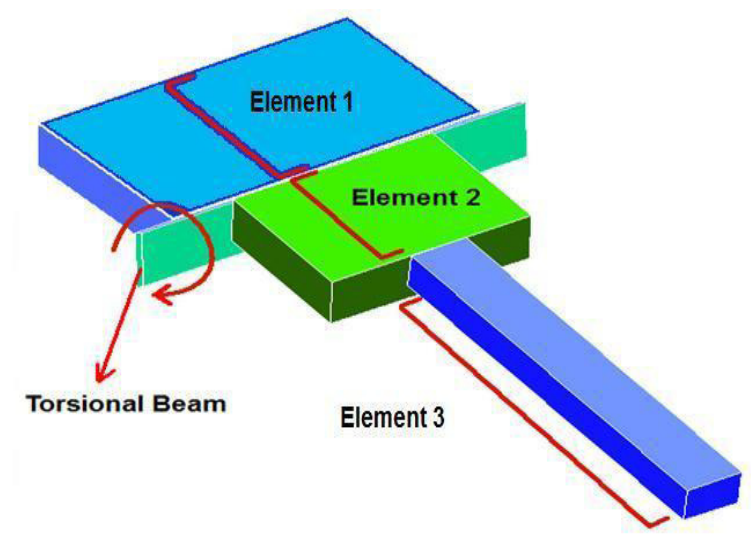

Fig. 2. Elements of the force sensor structure for FEM analysis.

to the fixed parts. Then, Modal Analysis is applied to find dominant natural frequencies and mode shape vectors. Also, an observer is necessary for estimating the applied velocity because the sensor is able only to measure displacement. Finally, an efficient closed loop system is developed to improve the performance of the sensor during manipulation by suppressing vertical disturbances.

\section{Modeling by finite element method}

Figure 1 illustrates a CAD model of the CMOSMEMS capacitive force sensor developed in Khir et al. [2]. In the proposed sensing mechanism, a micro probe is attached to the proof mass to convert the effect of applied vertical external force at the tip to produce relative displacement between the rotor (movable) and stator (fixed) comb drives and to capacitance changes during sensing.

To model the force sensor, finite element method is applied. The force sensor is considered as a beam composed of three different cross sections. As illustrated in Figure 2, the proof mass of the force sensor is anchored by two rotational beams. Consequently, three elements and four 
R.J. Mozhdehi et al.: Mechanics \& Industry 16, 306 (2015)

$$
K=\left[\begin{array}{ccccccc}
2.1751 \times 10^{5} & 27.1890 & 27.1890 & 0 & 0 & 0 & 0 \\
27.1890 & 761.2944 & 0.0034 & 0 & 0 & 0 & 0 \\
27.1890 & 0.0034 & 1.338 \times 10^{3} & -20.5977 & 0.0026 & 0 & 0 \\
0 & 0 & -20.5977 & 1.6746 \times 10^{5} & -19.1264 & -2.675 \times 10^{3} & 1.4713 \\
0 & 0 & 0.0026 & -19.1264 & 576.7390 & -1.4713 & 8.092 \times 10^{-4} \\
0 & 0 & 0 & -2.675 \times 10^{3} & -1.4713 & 2.675 \times 10^{3} & -1.4713 \\
0 & 0 & 0 & 1.4713 & 8.092 \times 10^{-4} & -1.4713 & 8.092 \times 10^{-4}
\end{array}\right]
$$

$$
\mathrm{M}=\mathrm{M}_{1}+\mathrm{M}_{2}
$$

$\mathrm{M}_{1}=$

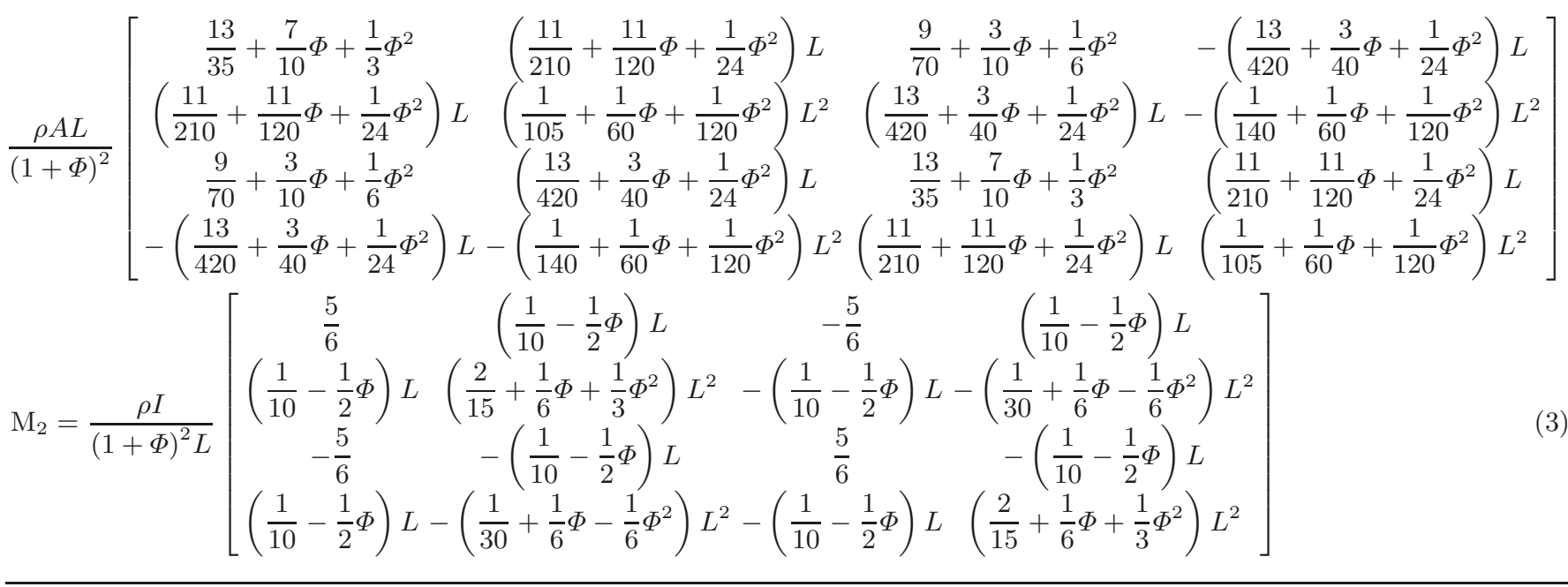

$\mathrm{M}=$

$\left[\begin{array}{ccccccc}7.4853 \times 10^{-9} & 2.4073 \times 10^{-13} & -1.4427 \times 10^{-13} & 0 & 0 & 0 & 0 \\ 2.4073 \times 10^{-13} & 1.123 \times 10^{-17} & -8.431 \times 10^{-18} & 0 & 0 & 0 & 0 \\ -1.4427 \times 10^{-13} & -8.431 \times 10^{-18} & 1.9737 \times 10^{-17} & 1.093 \times 10^{-13} & -6.3872 \times 10^{-18} & 0 & 0 \\ 0 & 0 & 1.093 \times 10^{-13} & 7.3131 \times 10^{-9} & 4.3583 \times 10^{-13} & 5.6547 \times 10^{-10} & -1.4987 \times 10^{-13} \\ 0 & 0 & -6.3872 \times 10^{-18-18} & 4.3583 \times 10^{-13} & 5.9267 \times 10^{-17} & 1.4987 \times 10^{-13}-3.8071 \times 10^{-17} \\ 0 & 0 & 0 & 5.6547 \times 10^{-10} & 1.4987 \times 10^{-13} & 1.6425 \times 10^{-9} & 2.5346 \times 10^{-13} \\ 0 & 0 & 0 & -1.4987 \times 10^{-13} & -3.8071 \times 10^{-17} & 2.5346 \times 10^{-13} & 5.076 \times 10^{-17}\end{array}\right]$

nodes should be considered. The dimensions of these elements are mentioned in Table 1. Timoshenko Beam model is considered and the stiffness matrix $(K)$ can be calculated for each element based in Equation (1) [15].

$$
K=\frac{E I}{L}\left[\begin{array}{cccc}
0 & 0 & 0 & 0 \\
0 & 1 & 0 & -1 \\
0 & 0 & 0 & 0 \\
0 & -1 & 0 & 1
\end{array}\right]+\frac{\mu G A}{4 L}\left[\begin{array}{cccc}
4 & 2 L & -4 & 2 L \\
2 L & L^{2} & -2 L & L^{2} \\
-4 & -2 L & 4 & -2 L \\
2 L & L^{2} & -2 L & L^{2}
\end{array}\right]
$$

where $E, L, G$ and $A$ are Young's modulus, length, shear modulus and cross action of each element respectively. Traditionally, $\mu$ is the correction factor for shear energy whose value is normally $\frac{5}{6}$. Assembling these elements, the stiffness matrix of the structure can be obtained and defined by Equation (2).

\section{See equation (2) above.}

The consistent mass matrix for Timoshenko Beam is applied to calculate the mass matrix of each element based in Equation (3) [15].

\section{See equation (3) above.}

where $\rho$ is the density of the structure and $\Phi=\frac{12 E I}{G A L^{2}}$. Finally assembling these elements, the mass matrix of the structure can be obtained as presented in Equation (4).

$$
\text { See equation (4) above. }
$$

Generally, the structural damping can be presented by Equation (5) [17].

$$
\mathrm{C}=\alpha \mathrm{M}+\beta \mathrm{K}
$$


Table 1. The dimensions of elements.

\begin{tabular}{cccc}
\hline Element & Length $(\mu \mathrm{m})$ & Width $(\mu \mathrm{m})$ & Thickness $(\mu \mathrm{m})$ \\
\hline 1 & 250 & 924 & 40 \\
2 & 250 & 700 & 40 \\
3 & 1100 & 50 & 40 \\
\hline
\end{tabular}

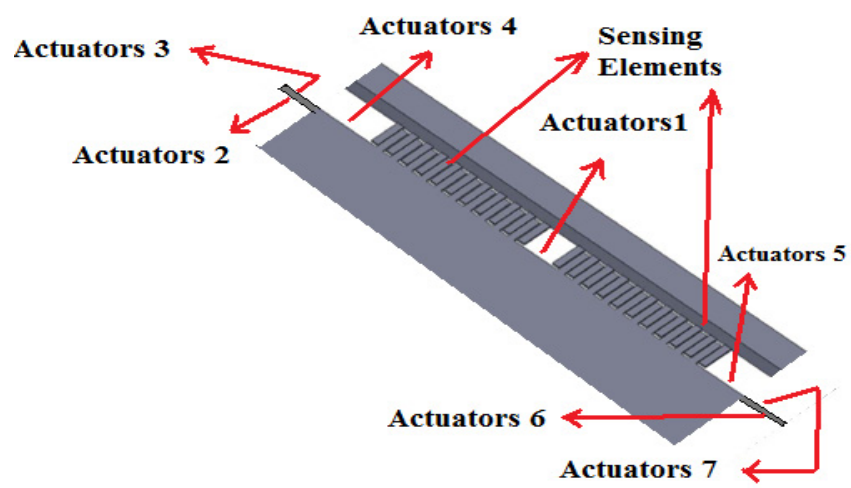

Fig. 3. Seven groups of electrostatic actuators located on the node No. 1.

The global equation of motion can be obtained employing Equation (6).

$$
[\mathrm{M}]\{\ddot{Y}\}+[\mathrm{C}]\{\dot{Y}\}+[\mathrm{K}]\{Y\}=\left[P_{C}\right]\{\mathrm{F}\}
$$

where $F$ is the force generated by electrostatic actuators against an undesired displacement, $P c$ is determined by the position of actuator and $Y$ is the vector of variables of nodes which was obtained as defined by Equation (7).

$$
Y=\left[\begin{array}{lllllll}
Y_{1} & \theta_{1} & \theta_{2} & Y_{3} & \theta_{3} & Y_{4} & \theta_{4}
\end{array}\right]
$$

Because the anchor is located on the node 2, Y2 should be removed. After determining the stiffness matrix, the magnitude of the force corresponding to each node can be calculated by Equation (8):

$$
F=K \times Y
$$

The comparison between the displacements estimated by FEM and the corresponding values from experimental model presented in Khir et al. [2] is given in Table 2. The results illustrate that the proposed model in this research is accurate enough for simulation study.

\section{Design of electrostatics actuators}

The node No. 1 is assigned for the attachment point of the electrostatic actuator because of more vacant space. Increasing the number of actuators causes to enhance the generated force with the same voltage. It can be supposed that two thin rods are added to the end of the sensor on the node No. 1 to make the possibility of increasing the number of actuators. Figure 3 illustrates the places of seven groups of electrostatic actuators located on the node No. 1.
In this case, the length of actuators should be selected as large as possible because of generating larger force with the same voltage. Consequently, it was selected $100 \mu \mathrm{m}$ which is the maximum length because of limitation of the space of the first actuators group. In addition, the efficient width of the actuator combs was selected as only $5 \mu \mathrm{m}$ so that the actuators fingers can be approximately located in parallel. Furthermore, the gap should be selected as small as possible due to generate larger force. So, the gap between movable and fixed actuator fingers can be selected as $5 \mu \mathrm{m}$ which is the minimum magnitude by considering the maximum displacement of the tip. Also, selecting smaller thickness can cause to increase the number of actuators and to generate larger force. Thus, the thickness was considered as $5 \mu \mathrm{m}$ an acceptable magnitude for tolerating forces. According to the dimensions set, it is reasonable for each of the seven groups to include two movable and three fixed electrostatic actuators. Consequently, 14 pairs of electrostatic actuators are suggested for applying the control algorithm. Figure 4 shows the structure of two actuators pairs of each group.

As illustrated in Figure 4, for each actuator, two surfaces, top and bottom, were defined to generate forces in different directions, one force is generated toward up and another is generated toward down. As illustrated in Figure 4, when the displacement generated by a disturbance is positive and the movable fingers move toward up, the positive controlling voltage should be applied to the top surfaces of the second and third fixed actuators and the bottom surfaces of both movable actuators because of generating forces toward down. This controlling voltage will be named "the controlling voltage for positive displacements" in the next sections. Similarly, for the negative value of the displacement measured by the sensor, the movable fingers move toward down and the positive controlling voltage should be applied to the bottom surfaces of the first and second fixed actuators and the top surfaces of both movable actuators because of generating forces toward up. This controlling voltage will be named "the controlling voltage for negative displacements" in the next sections. Both voltages will be applied by a controller continuously due to place the movable fingers accurately in the middle of the fixed fingers.

\section{Pull-in voltage}

For the system under investigation, the pull-in voltage is obtained when the mechanical force is equal to the electrical force [16]. Generally, to prevent damage and obtain high performance of the electrostatic devices they should be designed to operate at voltage below the pull-in voltage. In this case, when the pull-in voltage is applied, movable plate displaced by $1 / 3$ rd of the initial gap. Pull-in voltage can be calculated by Equation (9) [16].

$$
V_{\text {pull-in }}=\frac{2 X_{0}}{3} \sqrt{\frac{K_{0}}{1.5 C_{0}}}
$$


Table 2. Comparison between experimental and simulated models.

\begin{tabular}{cccl}
\hline Force $=100 \mathrm{nN}$ & Force $=500 \mathrm{nN}$ & Force $=1 \mu \mathrm{N}$ & \\
\hline $5.27 \times 10^{-8}$ & $2.62 \times 10^{-7}$ & $5.27 \times 10^{-7}$ & Displacement of the end of sensing finger in Experimental Model \\
$5.2644 \times 10^{-8}$ & $2.6172 \times 10^{-7}$ & $5.2644 \times 10^{-7}$ & Displacement of the end of sensing finger in Simulated Model by FEM \\
\hline
\end{tabular}

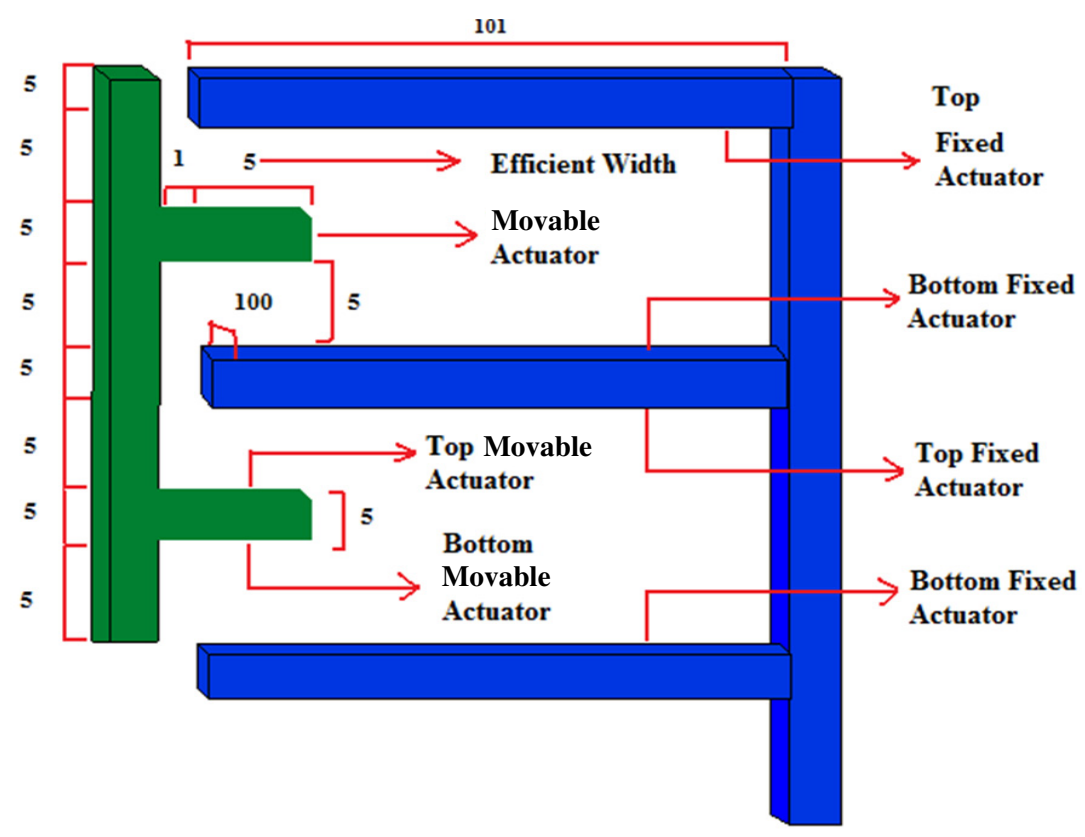

Fig. 4. The structure of two actuators pairs of each group.

where $X_{0}$ is given by Equation (10).

$$
X_{0}=g+d_{\max }
$$

in which $g$ represents the gap between movable and fixed plate and it was determined to be equal to $5 \mu \mathrm{m}$. In addition, $d_{\max }$ is equal to possible maximum displacement. As mentioned in Khir et al. [2], the maximum measurable force by the sensor under investigation is up to $1 \mu \mathrm{N}$. The movable part of the sensor usually faces with disturbance forces generated by eventually clashing a cell or tissue in nano scale when it is applied in living cell manipulation or minimally invasive surgery and more powerful disturbance forces usually apply to arm of manipulator which should be damped by the arm. Accordingly, the sensor is appropriate for damping disturbance forces applied to the movable part of the sensor and maximum disturbance force supposed to be $1 \mu \mathrm{N}$ which generates a displacement equal to $0.539 \mu \mathrm{m}$ in node No. 1 based in Equation (8). Also $K_{0}$ can be calculated by Equation (11):

$$
K_{0}=\frac{F_{0}}{X_{0}}
$$

In this equation, $F_{0}$ is the force which can generate $d_{\text {max }}$ based in Equation (8) and $C_{0}$ can be calculated by Equation (12):

$$
C_{0}=\frac{\varepsilon L w}{X_{0}}
$$

where, $L$ and $w$ are the efficient length and the width of actuators respectively. Based in Equations (8)-(12) the pull-in voltage was calculated as $85.72 \mathrm{~V}$.

\section{Modal analysis}

Modal analysis adheres that only the first few modes are significant for obtaining the vibration response. Consequently, an approximate reduced order dynamic model of the structure is considered in modal coordinates based in Equation (13) [17].

$$
\{Y\}=[\hat{\Phi}]\{\eta\}
$$

where $\{\eta(t)\}$ are the modal coordinates and $[\hat{\Phi}]$ is the truncated modal matrix which can be calculated by Equation (14).

$$
[\hat{\Phi}]=\left[\Phi_{1}, \Phi_{2}, \ldots, \Phi_{r}\right]
$$

In this equation, $\Phi_{r}$ is the shape mode vector of $r$ th frequency which is normalized employing Equations (15) and (16).

$$
\begin{aligned}
& \Phi_{r}^{T} \mathrm{M} \Phi_{r}=1 \\
& \Phi_{r}^{T} \mathrm{~K} \Phi_{r}=\omega_{r}^{2}
\end{aligned}
$$


Based on modal coordinates, the equation of motion calculated in Equation (6) could be transformed to the reduced modal space form as mentioned in Equation (17) [17].

$$
\left[\mathrm{M}^{\prime}\right]\{\ddot{\eta}\}+\left[\mathrm{C}^{\prime}\right]\{\dot{\eta}\}+\left[\mathrm{K}^{\prime}\right]\{\eta\}=\left[\mathrm{P}_{\mathrm{C}}^{\prime}\right]\{F\}
$$

where $\left[\mathrm{M}^{\prime}\right],\left[\mathrm{C}^{\prime},\left[\mathrm{K}^{\prime}\right]\right.$, and $\left[\mathrm{P}_{\mathrm{C}}^{\prime}\right]$ are calculated by Equations $(18)-(21)$.

$$
\begin{aligned}
{\left[\mathrm{M}^{\prime}\right] } & =[\hat{\Phi}][\mathrm{M}][\hat{\Phi}] \\
{\left[\mathrm{C}^{\prime}\right] } & =[\hat{\Phi}][\mathrm{C}][\hat{\Phi}] \\
{\left[\mathrm{K}^{\prime}\right] } & =[\hat{\Phi}][\mathrm{K}][\hat{\Phi}] \\
{\left[\mathrm{P}_{\mathrm{C}}^{\prime}\right] } & =[\hat{\Phi}]\left[\mathrm{P}_{\mathrm{C}}\right]
\end{aligned}
$$

Then, the equation of motion can be written in a statespace as presented by Equations (22) and (23).

$$
\begin{aligned}
{[\xi] } & =\left\{\begin{array}{l}
\eta \\
\dot{\eta}
\end{array}\right. \\
{[\dot{\xi}] } & =[\mathrm{A}]\{\xi\}+[\mathrm{B}]\{u\}
\end{aligned}
$$

where $[\mathrm{A}]$ and $[\mathrm{B}]$ are the system and control matrixes, respectively as mentioned by Equations (24) and (25).

$$
\begin{aligned}
& {[\mathrm{A}]=\left[\begin{array}{cc}
{[0]} & {[I]} \\
-\left[\mathrm{M}^{\prime}\right]\left[\mathrm{K}^{\prime}\right] & -\left[\mathrm{M}^{\prime}\right]\left[\mathrm{C}^{\prime}\right]
\end{array}\right] .} \\
& {[\mathrm{B}]=\left[\begin{array}{c}
{[0]} \\
-\left[\mathrm{M}^{\prime}\right]\left[\mathrm{P}_{\mathrm{C}}^{\prime}\right]
\end{array}\right]}
\end{aligned}
$$

where $\{u\}=F(t)$ is the controlling force.

\section{Optimal control law and minimum-order observer}

To generate the controlling force, the gains of a controller may be determined by optimal control law and named linear quadratic regulator (LQR) controller. After simulating the Force Sensor, LQR controller can be designed by MATLAB software. The most significant point of designing LQR controller is that $\mathrm{Q}$ and $\mathrm{R}$ have to be calculated by respecting pull-in voltage and acceptable controlling time.

Generally, electrostatic actuators cannot be directly controlled by this control algorithm. The reason is that the controller generates force signals while electrostatic actuators require voltage or current signals to be operated. To solve the problem, we use an inverse electrostatic actuators model and force can be calculated by Equation (26) [18].

$$
F=\frac{\varepsilon L w}{2(g+d)^{2}} V^{2}
$$

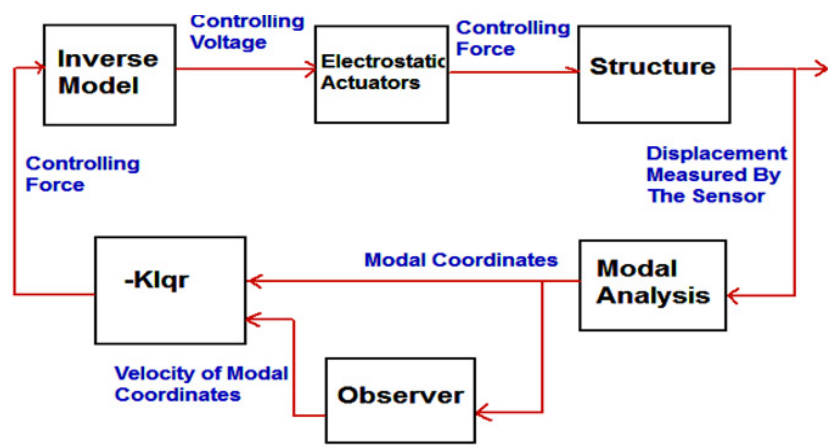

Fig. 5. The block diagram of the control algorithm.

where $d$ is the displacement and $g$ is the gap between the movable and fixed plates. In this way, we can obtain the controlling voltage which should be applied to the actuators. Figure 5 illustrates the block diagram of the control algorithm computed with inverse electrostatic actuators model. As shown in this figure, the control algorithm needs an observer for estimating the velocity of the modal coordinates.

It should be noted that some of the variables cannot be measured. On the other hand, the displacement can be measured by the sensor and the modal coordinate can be calculated by the displacement but, velocity of the modal coordinate cannot be measured. Consequently, a minimum-order observer is needed for estimating them based on measured displacement and modal coordinate. First of all, the controllability matrix should be investigated based in Equation (27) [19]:

$$
\mathrm{M}=\left[\mathrm{B}|\mathrm{AB}| \ldots \mid \mathrm{A}^{\mathrm{n}-1} \mathrm{~B}\right]
$$

where $\mathrm{n}$ is the number of the states. The partitioned states can be presented by Equation (28) [20].

$$
\left[\begin{array}{c}
\dot{\xi_{a}} \\
\overline{\dot{\xi}_{b}}
\end{array}\right]=\left[\begin{array}{lll}
A_{a a} & \mid & A_{a b} \\
\overline{A_{b a}} & - & \overline{A_{b b}}
\end{array}\right] \quad\left[\begin{array}{c}
\xi_{\mathrm{a}} \\
\overline{\xi_{\mathrm{b}}}
\end{array}\right]+\left[\begin{array}{c}
B_{a} \\
\overline{B_{b}}
\end{array}\right] u
$$

where $\xi_{a}$ is a scalar and can be directly measured. $\xi_{b}$ is $(n-1) \times 1$ matrix and is the immeasurable portion of the state vector. $A_{a a}, A_{a b}, A_{b a}, A_{b b}, B_{a}, B_{b}$ are scalar, $1 \times(n-1)$ matrix, $(n-1) \times 1$ matrix, $(n-1) \times(n-1)$ matrix, scalar and $(n-1) \times 1$ matrix respectively. Based in Equation (28), the gains of the minimum-order observer can be determined by MATLAB software. It is necessary to properly propose the eigenvalues of minimum-order observer. Then, transfer function of the observer-controller should be investigated and if it includes a positive eigenvalue, the eigenvalue of minimum-order observer should be changed.

\section{Simulation study and results}

The natural frequencies of the system can be obtained by solving the eigenvalue problem of the generalised $\left(\mathrm{M}^{-1} \times \mathrm{K}\right)$, the natural frequencies can be obtained. 
Table 3. The natural frequencies.

\begin{tabular}{ccccccc}
\hline$\omega 1$ & $\omega 2$ & $\omega 3$ & $\omega 4$ & $\omega 5$ & $\omega 6$ & $\omega 7$ \\
\hline $9.0800 \times 10^{+9}$ & $4.6516 \times 10^{+9}$ & $9.7961 \times 10^{+8}$ & $2.1370 \times 10^{+7}$ & $5.3906 \times 10^{+6}$ & $3.2673 \times 10^{+6}$ & $2.6604 \times 10^{+4}$ \\
\hline
\end{tabular}

Table 4. The gains of observer-controller.

\begin{tabular}{lc}
\hline LQR gain $K(1,1)$ & $8.9103 \times 10^{-10}$ \\
LQR gain $K(1,2)$ & $98393 \times 10^{-4}$ \\
Observer gain Ke & $2.1602 \times 10^{+4}$ \\
\hline
\end{tabular}

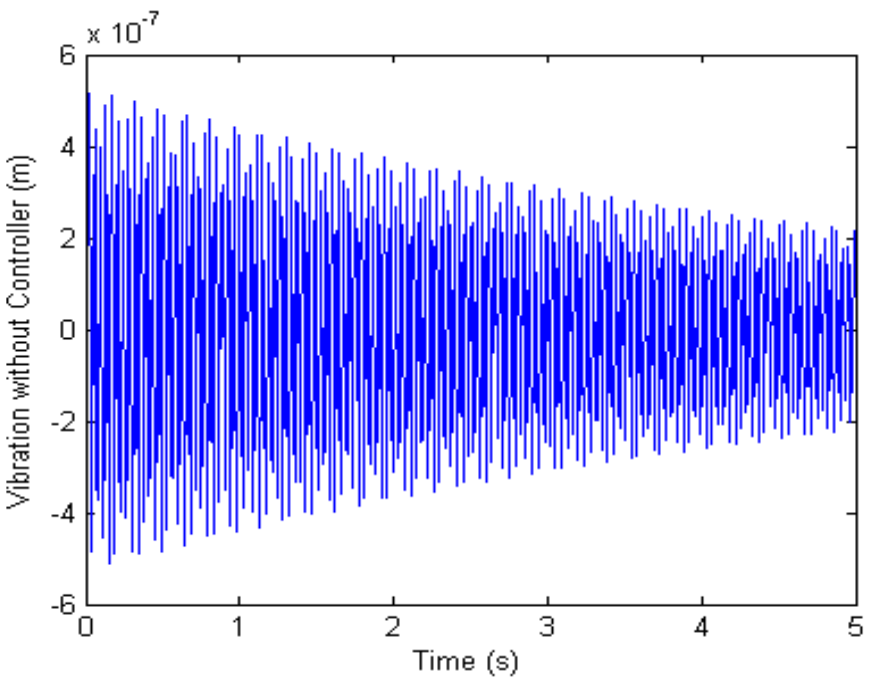

Fig. 6. Open loop vibration of the sensor for the maximum displacement.

They are listed in Table 3. The first mode was considered since it is critical in vibration analysis. Based in Equations (13)-(25), the new equations of motion and state space equations related to modal coordinate were achieved. As mentioned, the maximum displacement of the end of the probe is equal to $0.539 \mu \mathrm{m}$. Figure 6 illustrates open loop vibration response for the maximum displacement of the probe. As understood from the Equation (29), the new state space equations are controllable because the rank of controllability matrix $(\mathrm{CM})$ is equal with the rank of matrix $\mathrm{A}$ which is 2 .

$$
\mathrm{CM}=[\mathrm{B} \quad \mathrm{AB}]
$$

Then, LQR algorithm was implemented using MATLAB software and selecting $Q=\left[\begin{array}{ll}1 & 0 \\ 0 & 1\end{array}\right]$ and $R=10000000$. We obtained two possible values for controlling gain. In addition, using MATLAB software, the gain of the minimumorder observer was determined. The gains of the observercontroller are presented in Table 4.

Then, transfer function of the observer-controller was calculated and as understood from Equation (30), all of its eigenvalues are negative.

$$
G_{e s}=\frac{6.1121 s-1.4170 \times 10^{12}}{s+2.1920 \times 10^{3}}
$$

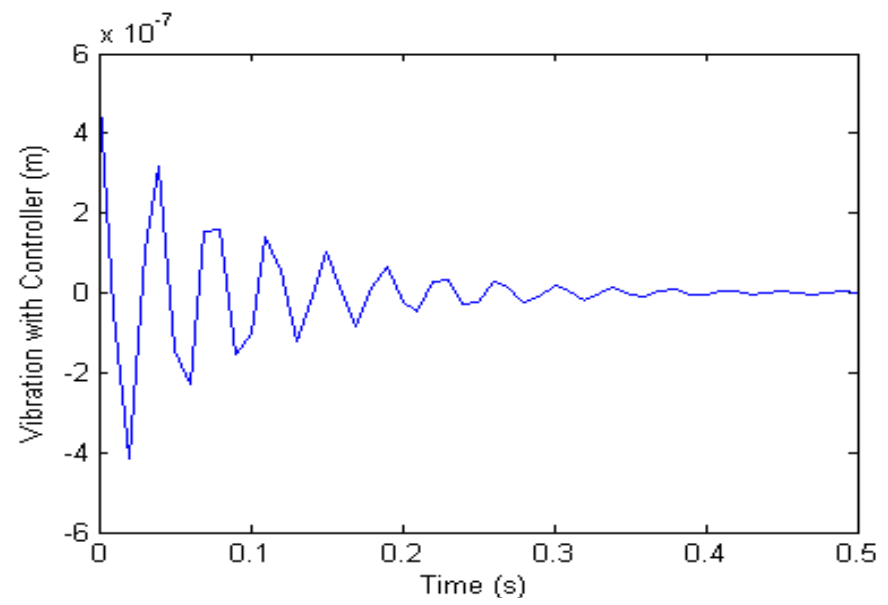

Fig. 7. Closed loop vibration of the sensor structure for the maximum displacement.

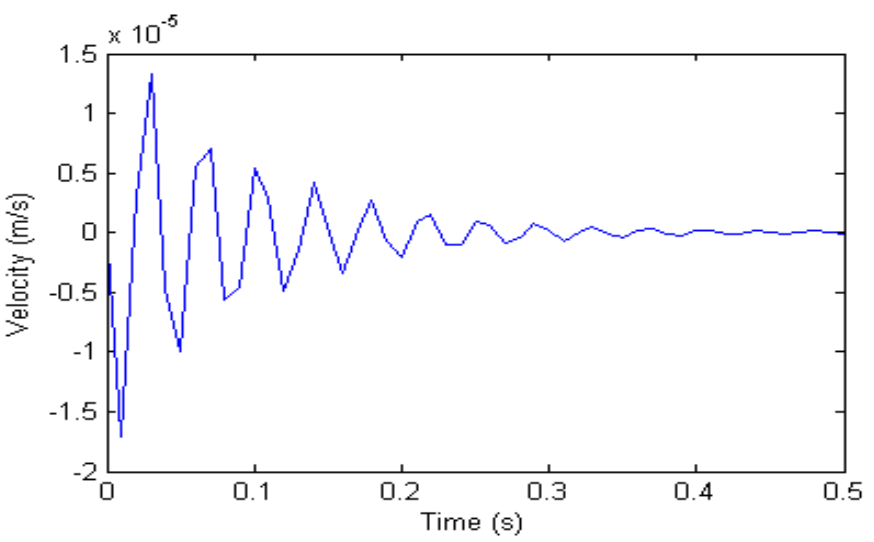

Fig. 8. The velocity of the modal coordinate estimated by the minimum-order observer.

Closed loop response of the system employing the observer-controller for the maximum disturbance input is simulated and illustrated in Figure 7. Employing the proposed control algorithm, the maximum disturbance input is suppressed less than $0.4 \mathrm{~s}$ as shown in Figure 7. Additionally, the velocity of the modal coordinate was estimated by the minimum-order observer and illustrated in Figure 8.

The controlling forces needed for suppressing the maximum disturbance are illustrated in Figure 9. Based on the controlling force and using inverse model calculated by Equation (26), the controlling voltages which should be applied to actuators can be calculated. In addition, Figures 10 and 11 illustrate the controlling voltages applied to the electrostatic actuators to suppress the maximum disturbance of the probe. For instance, as shown in Figure $1,3.5 \mathrm{~V}$ was applied to the top surfaces of the second and third fixed actuators and the bottom surfaces of both 


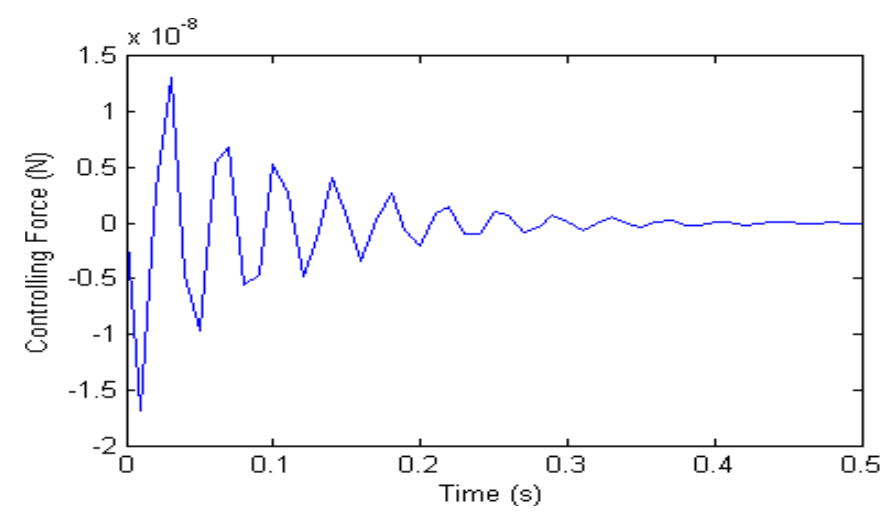

Fig. 9. The controlling forces needed for suppressing the maximum displacement.

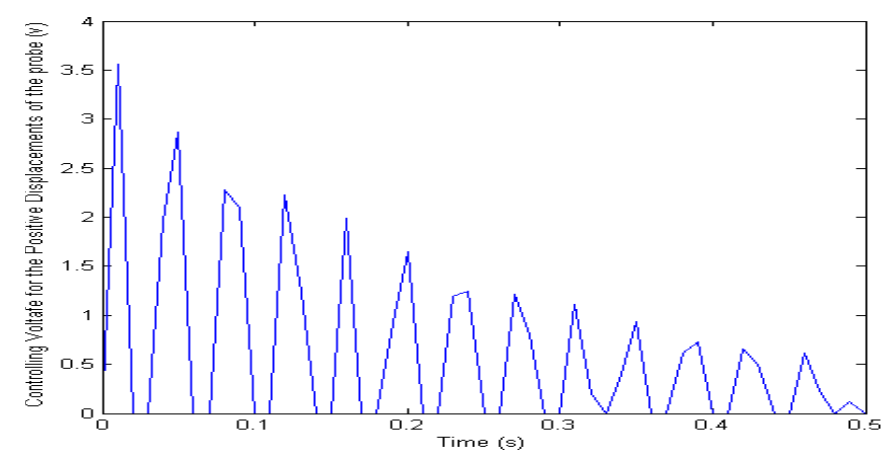

Fig. 10. The controlling voltage for positive displacements of the probe.

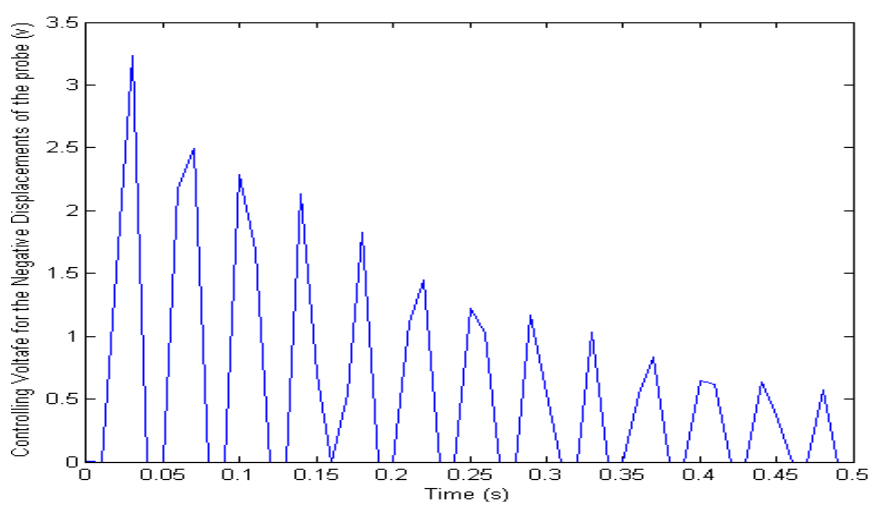

Fig. 11. The controlling voltage for negative displacements of the probe.

movable actuators due to suppress the positive displacement. Then, the sensor measured the negative displacement and $3.1 \mathrm{~V}$ was applied to the bottom surfaces of the first and second fixed actuators and the top surfaces of both movable actuators because of generating forces toward up. It is understood that the maximum controlling voltage $(3.5 \mathrm{~V})$ for suppressing the maximum disturbance which should be applied to top electrostatic actuators is less than pull-in voltage $(85.72 \mathrm{~V})$ calculated by Equation (9).

\section{Conclusion}

This paper has addressed the performance of a new CMOS-MEMS nano-newton capacitive force sensor using a finite element method technique. The necessary modification needed to improve the accuracy of the proposed model was performed employing comparison between the results of simulation and experimental analysis. In this case, 14 pairs of electrostatic actuators which were categorized in seven groups were designed to suppress vertical disturbances generated by environment during manipulation. In addition, pull-in voltage needed to actuate the actuators was calculated. Finally an optimal controller was proposed utilizing the state-space and modal analysis approaches. Modal analysis was applied to find dominant natural frequencies and mode shape vectors. Also, the velocity of the modal coordinate was estimated by an observer. Simulation results illustrate that by employing optimum LQR control approach, the maximum disturbance input is suppressed less than $0.4 \mathrm{~s}$. The corresponding maximum controlling voltage is obtained as $3.5 \mathrm{~V}$ which should be applied to the electrostatic actuators located on top which is less than pull-in voltage $(85.72 \mathrm{~V})$.

\section{References}

[1] M.G. Susan, et al., A Submersible Piezoresistive MEMS Lateral Force Sensor for a Diagnostic Biomechanics Platform, Sensors and Actuators 188 (2012) 111-119

[2] M.H. Khir, et al., A CMOS-MEMS Nano-Newton Force Sensor for Biomedical Applications. In: Proc. 5th IEEE International Nano/Micro Engineered and Molecular Systems Conference, 2010

[3] P. Estevez, et al., 6 DOF force and torque sensor for micro-manipulation applications, Sensors and Actuators 186 (2012) 86-93

[4] V. Kutis, et al., MEMS piezoelectric pressure sensor modelling and simulation, Proc. Eng. 48 (2012) 338-345

[5] Y. Ge, et al., An Optical MEMS Pressure Sensor based on a Phase Demodulation Method, Sensors and Actuators 143 (2012) 224-229

[6] J. Wei, et al., Suspended submicron silicon-beam for high sensitivity piezoresistive force sensing cantilevers, Sensors and Actuators 186 (2012) 80-85

[7] H.K. Chu, et al., Design of a High Sensitivity Capacitive Force sensor, In: Proc. 7th IEEE Nanotechnology International Conference, 2007

[8] H. Xie, G.K. Fedder, A CMOS $z$-axis capacitive accelerometer with comb-finger sensing, In: Proc. 13th International MEMS Conference, 2000

[9] M. Tsai, et al., A CMOS-MEMS Accelerometer with Triaxis Sensing Electrodes Arrays, Proc. Eng. 5 (2010) 10831086

[10] H. Xie, G.K. Fedder, Vertical comb-fingers capacitive actuation and sensing for CMOS-MEMS, Sensors and Actuators 95 (2002) 212-221

[11] S.M. Kumar, M.V. Lakshmi, Shape Memory Alloys and its Application in MEMS Devices, Current Eng. Technol. 3 (2013) 
[12] P.A. Gedouin, et al., Experimental Comparison of Classical PID and Model-Free Control on Position Control of a Shape Memory Alloy Active Spring, Control Engineering Practice 19 (2011) 433-441

[13] M. Marinaki, et al., Vibration Control of Beams with Piezoelectric Sensors and Actuators using Particle Swarm Optimization, Expert Syst. Appl. 38 (2011) 6872-6883

[14] K.H. Koha, et al., Investigation of a Piezoelectric Driven MEMS Mirror based on Single S-shaped PZT Actuator, Proc. Eng. 25 (2011) 701-704

[15] Y.W. Kwon, H. Bang, The Finite Element Method Using MATLAB, CRC Press, USA, 1997, pp. 235-242
[16] V. Balamurugan, S. Narayanan, Finite Element Formulation and Active Vibration Control Study on Beams Using Smart Constrained Layer Damping (SCLD) Treatment, Sound Vib. 249 (2002) 227-250

[17] C. Liu, Foundations of MEMS, Prentice Hall Press Upper Saddle River, USA, 2011

[18] T. Hsu, Mems and Microsystems, John Wiley \& Sons, USA, 2007

[19] M. Gopal, Modern Control System Theory, New Age International, India, 1993

[20] K. Ogata, Modern Control Engineering, Hall Press Upper Saddle River, USA, 2011, pp. 15-925 